\title{
Mixture Modeling based Multikernel Sparse Learning for Directional of Arrival Estimation
}

\author{
Awab Habib Fakih, S M Shashidhar
}

\begin{abstract}
Direction of Arrival (DOA) estimation problem is defined as the problem of Sparse Signal Recovery (SSR) in researches published on the Uniform or Non Uniform array based implementations. This Paper attempts a Multikernel Sparse learning (MSL) approach with mixture modeling for the $S S R$ problem to improve the performance parameters including the PSNR and the RMSE of the estimated sparse signal in the underdetermined condition. The Expectation Maximization algorithm is exploited to obtain the convergence in the mixture modeling MSL method. The virtual array response problem thus developed uses the mixture modeling MSL to estimate the DOA. Matlab based implementation is carried out and the results are found to be satisfactory.
\end{abstract}

Keywords : About four key words or phrases in alphabetical order, separated by commas.

\section{INTRODUCTION}

Nowadays DOA estimation methods with higher Degree of Freedom (DOF) with less number of sensor by constructing a virtual array method is implemented with advanced SSR techniques introduced[1]. The mixture model would be more flexible when dealing with multiple heterogeneous sources [2-3]. Numerous model selection problems are dealt using Sparse Bayesian regression models by creating the sparse prior for the model parameters [4-7]. The Sparse Bayesian regression models are able to obtain sparse models with higher generalization thus obtaining a better accuracy in SSR problems [1]. As the extension of the SBL models the RVM models are surfaced recently. The literature [6] deals about a weighted multikernel RVM [8] model where multiple kernels are combined by weighing them while weight values are estimated during training. The virtual array is developed to populate different Angle of Arrival (AOA) is utilized for the DOA estimation problem, meaning increasing the DOF without increasing the sensors as discussed in literature [9]. Numerous implementations with SBL methods for solving SSR problems are discussed in [10-14].

This Paper implements the mixture model MSL for modeling the SSR with a priori for parameter estimation and posterior expectation maximization algorithm for solving the mixture model RVM for DOA estimation.

\section{Problem Formulation for DOA estimation:}

Revised Manuscript Received on July 22, 2019.

Awab Habib Fakih , PhD Reserach Scholar, Visvesvaraya Technological University, Belagavi

Dr. S M Shashidhar, Principal, Proudhadeveraya Institute of Technology - [PDIT], Hospet
The DOA estimation problem starts with developing the source and the antenna structure along with the

type of antenna array. The structure involves the linear array of sensors that are placed non-uniformly for receiving the arriving signals from omnidirectional signals. $M$ number of such sensors are utilized with their distances from the sensor that acts as the reference at location 0 denoted as $\left[0, d_{1, \ldots \ldots,} d_{M-1}\right]$. This distance must be in terms of integral multiples of half the wavelength in order to have positive interference. The implementation carried out in [1] uses the virtual array to define the DOFs.

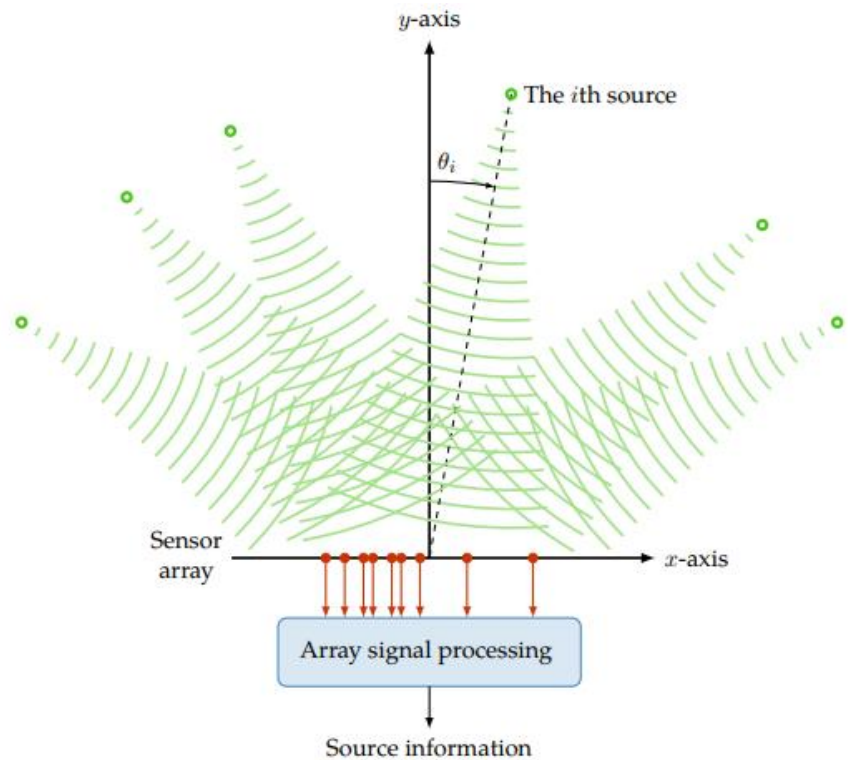

Figure 1. The Overall Structure of the Non Uniformly Placed Sensors[15]

In [9] the difference co- array or the virtual array from the sparse array is used for DOA estimation, which is defined by using the set of difference between the positions of the sensors denoted by $\Omega=\left\{d_{m 1}-d_{m 2}\right\}_{m 1}=0,1_{m, M-1 ; m 2}=0,1_{m-M-1}$. Since the implementation increases the DOFs the sparse array is denoted by $\Omega$, which has more than the number of sensors $M$. The $K$ number of uncorrelated narrowband far field sources is considered to be directed towards the sensor array, which is corrupted by the white Gaussian noise denoted by, $n_{m}(t), m=0,1_{, \ldots \ldots} M-1$. The $K$ sources with T snapshot $s_{k}(t), k=1,2, \ldots \ldots$ and the array output data is related as the 
following ,

$x(t)=A s(t)+n(t), t=1,2, \ldots \ldots x$

Array output data $x(t)$ is the addition of snapshots of the source incident on the array and the noise added at the $t^{\text {th }}$ snapshot. The matrix ' $A$ ' is the set of all steering vectors that would move in the directions of the virtual arrays that would be used to find the convergence. A matrix known as the manifold matrix is denoted as $A=\left[a\left(\theta_{1}\right), a\left(\theta_{2}\right), \ldots \ldots a\left(\theta_{K}\right)\right.$ which is of $K$ length, while the steering vector defined as, $a(\theta)=\left[1_{v} v\left(d_{1}, \theta\right), \ldots \ldots, v\left(d_{M-1}, \theta\right)\right]^{T}$ is the group of phase factors on the source impinging on the array where $\left[x^{T}\right\}^{T}$ the transpose is. The element in the steering vector called as the phase factor is given as, $v\left(d_{m}, \theta\right)=\exp \left[-j 2 \pi\left(\frac{d_{m}}{\lambda}\right) \sin \theta\right]$

The array output vector's covariance matrix with source and noise signals uncorrelated is given as

$$
R_{x}=E\left\{x(t) x^{H}(t)\right\}=\operatorname{Adiag}\left(\sigma_{1}^{2}, \sigma_{2}^{2}, \ldots \ldots \ldots \sigma_{R}^{2}\right) A^{H}+
$$

$\sigma_{n}^{2} I_{\mathbb{M}^{x}} \quad$ (2)

where $\mathrm{E}\{$.$\} is the expectation and I_{M}$ is an $M X M$ identity matrix where the variance $K$ for sources $\sigma_{1}^{2}, \sigma_{2}^{2}, \ldots \ldots \ldots \sigma_{K}^{2}$ the variance of noise $\sigma_{n}^{2}$. The vectorised $R_{x}$ is used [16]

$$
\begin{aligned}
& y=\operatorname{vec}\left(R_{x}\right)=\operatorname{vec}\left(A R_{S} A^{H}\right)+\sigma_{n}^{2} \operatorname{vec}(I)=\left(A^{*} \odot A\right) g+ \\
& \sigma_{n}^{2} 1_{M}
\end{aligned}
$$

The manifold matrix with the virtual array ${ }^{\prime} \bar{A}$ is the combination of $K$ different steering vectors which is the Kramer Roa (@) product of ${ }^{I} A^{* t}$ and ${ }^{\prime} A^{t}$ where ${ }^{\prime} A^{* t}$ is the conjugate of manifold matrix $A$. And the term $A^{H}$ is the conjugate transpose of $A$. The vector of source variances is $g=\sigma_{1}^{2}, \sigma_{2, \ldots \ldots \ldots}^{2} \sigma_{K}^{2}$ and the vector $1_{M}$ is defined as $\left[e_{1}^{T}, e_{2}^{T}, e_{2}^{T} \ldots, e_{M^{s}}^{T}\right]^{T} \quad$ with the term $e_{\mathrm{m}}$ with zeros everywhere while $\mathrm{m}^{\text {th }}$ position being 1 .

The steering vectors of the $\bar{A}$ matrix is defined by the array of vectors defined as, $\bar{a}\left(\theta_{k}\right)=a^{*}\left(\theta_{k}\right) \otimes a\left(\theta_{k}\right), k=1,2, \ldots . K$ , which is nothing but the $\operatorname{kronecker}(\Theta)$ product of the steering vector and its conjugate. These products are the distinct vectors that define the DOFs of the solution.

While the signal is available only the sample covariance matrix can be obtained, which is defined as,

$\hat{R_{x}}=\sum_{t=1}^{T} x(t) x^{H}(t) / T$

While the source signals that are incident on the antennas are considered to be Gaussian distributed with circular symmetry then the covariance matrix's error estimation for residuals is said to satisfy asymptotic complex Gaussian distribution as discussed in [17].

$$
\hat{y}-y=\operatorname{vec}\left(\hat{R}_{x}-\operatorname{vec}\left(R_{x}\right) \sim \operatorname{CN}\left(0, \frac{1}{T} R_{x}^{T} \otimes R_{x}\right)\right.
$$

The term $\frac{1}{\tau} R_{x}^{T} \otimes R_{x}$ be denoted as $\tilde{R}_{x}$ and applying (1) on (2) it gives

$$
\vec{y} \sim \operatorname{CN}\left(\bar{A} g+\sigma_{n}^{2} 1_{M^{*}} \tilde{R}_{x}\right)
$$

This equation (3) is the Basis Pursuit Denoising (BPDN) problem in order to develop the DOA estimation. With the idea to express (3) sparsely the sampling grid is formed using the different Angles of Arrival (AOA) of the source signals .This sampling grid defined as $\Theta=\left\{\theta_{1}, \theta_{2} \ldots \ldots, \theta_{N}\right\}$ denotes the span of the AOA. Thus all the DOAs that can be estimated is characterized by applying the dense direction grid on the equation (3) and defined as shown in (4)

$$
\hat{y} \sim C \mathrm{CN}\left(\Phi w+\sigma_{n}^{2} 1_{M^{\prime}}, \hat{R}_{x}\right) \ldots \ldots
$$

If $\Theta$ acts as the dense grid the $\Phi$ is the set of all basis vectors in the grid for the virtual array manifold matrix $\bar{A}$ and non-negative sparse vector is the vector where the entries are zeros except the position where the actual DOAs are present.

\section{RESULTS AND DISCUSSION}

Mixed Model Multikernel Modeling is developed on this BPDN problem and the Matlab based simulation is carried out. The prior and the posterior distribution as defined in [18] for the problem defined in the above section is developed and the results are obtained as below. The structure of the antenna that is defined for the implementation as given in the following Table1.

Table1. The Antenna Configuration for the proposed SSR implementation

\begin{tabular}{|c|c|}
\hline Details & Configuration \\
\hline $\begin{array}{c}\text { Number of } \\
\text { Antennas }\end{array}$ & 4 \\
\hline Antenna Array type & Non-uniform \\
\hline Angle Range & $-\frac{\pi}{2}$ to $\frac{\pi}{2}$ \\
\hline $\begin{array}{c}\text { Min to Max degrees } \\
\text { Carrier frequency }\end{array}$ & -70 to 70 \\
\hline $\begin{array}{c}\text { Propagation velocity } \\
\text { Interval of angle } \\
\text { Searching }\end{array}$ & $200 \mathrm{~Hz}$ \\
\hline $\begin{array}{c}\text { Angles of source } \\
\text { Signals }\end{array}$ & $\begin{array}{c}\text { Bublished By: } \\
\text { \& Syes Intelligence Engineering } \\
\text { Sciences Publication }\end{array}$ \\
\hline \\
\hline
\end{tabular}


The results for the DOA estimation of the proposed method are as given below. The results from the implementation would define the performance of the underdetermined conditions with the AOA as defined in the above table.

The experiment include the SSR implementation for DOA estimation for the structure configured as given in the Table1 and also the RMSE for the DOA estimation when the iteration is happening in order define the convergence of the algorithm. The Signal to Noise ratio is set for different values of noise and the corresponding snapshots are defined. It is found that the DOA estimation is found to be efficient compared to the previous implementation [19].
The four steering vector generated to form the manifold matrix $A$ is as shown in the following Figure 2. This manifold matrix is interpolated with the six source signals to get the different vectors that would span the $2 \mathrm{D}$ planar. The source Signals from different sources are depicted as discussed in Figure 3.

The source signal combined with the steering vector is as shown in Figure 4. The noise signal to be added with the source signal is shown in the Figure 5. The incident signal added with the noise is as shown in Figure 6.

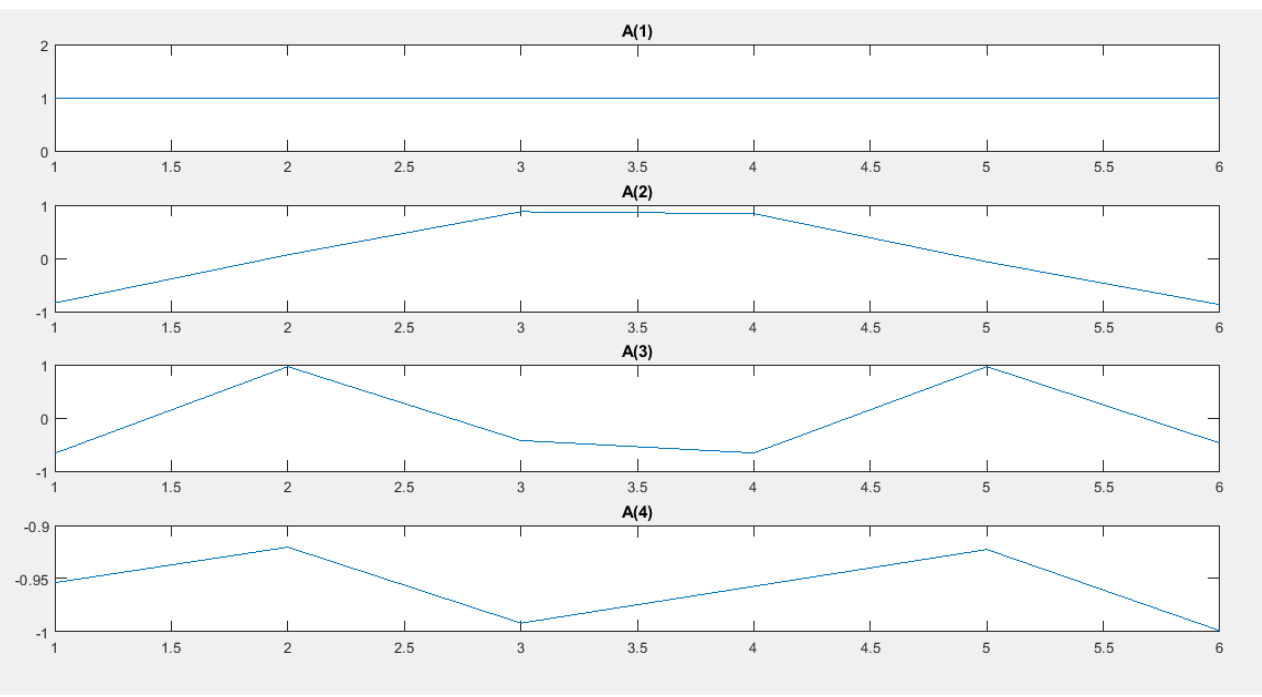

Figure 2.Steering Vectors of the Manifold matrix

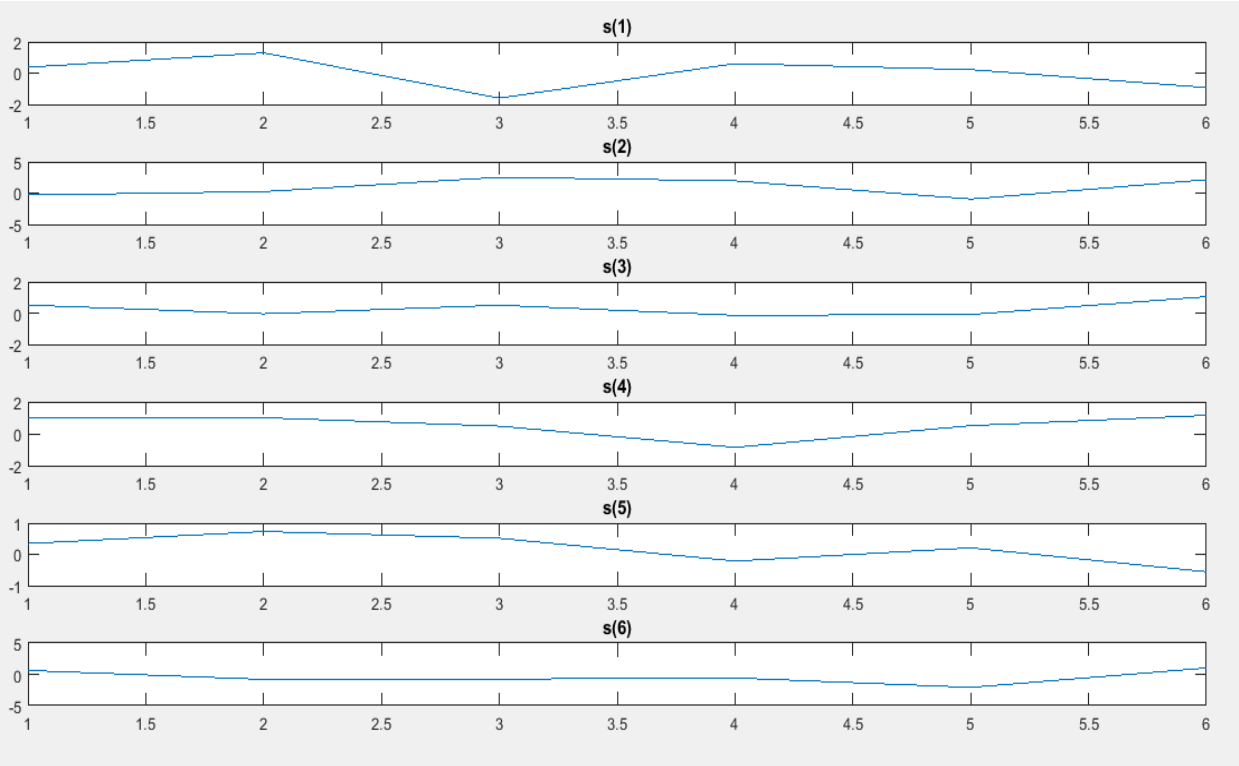

Figure 3. Incident Signals 

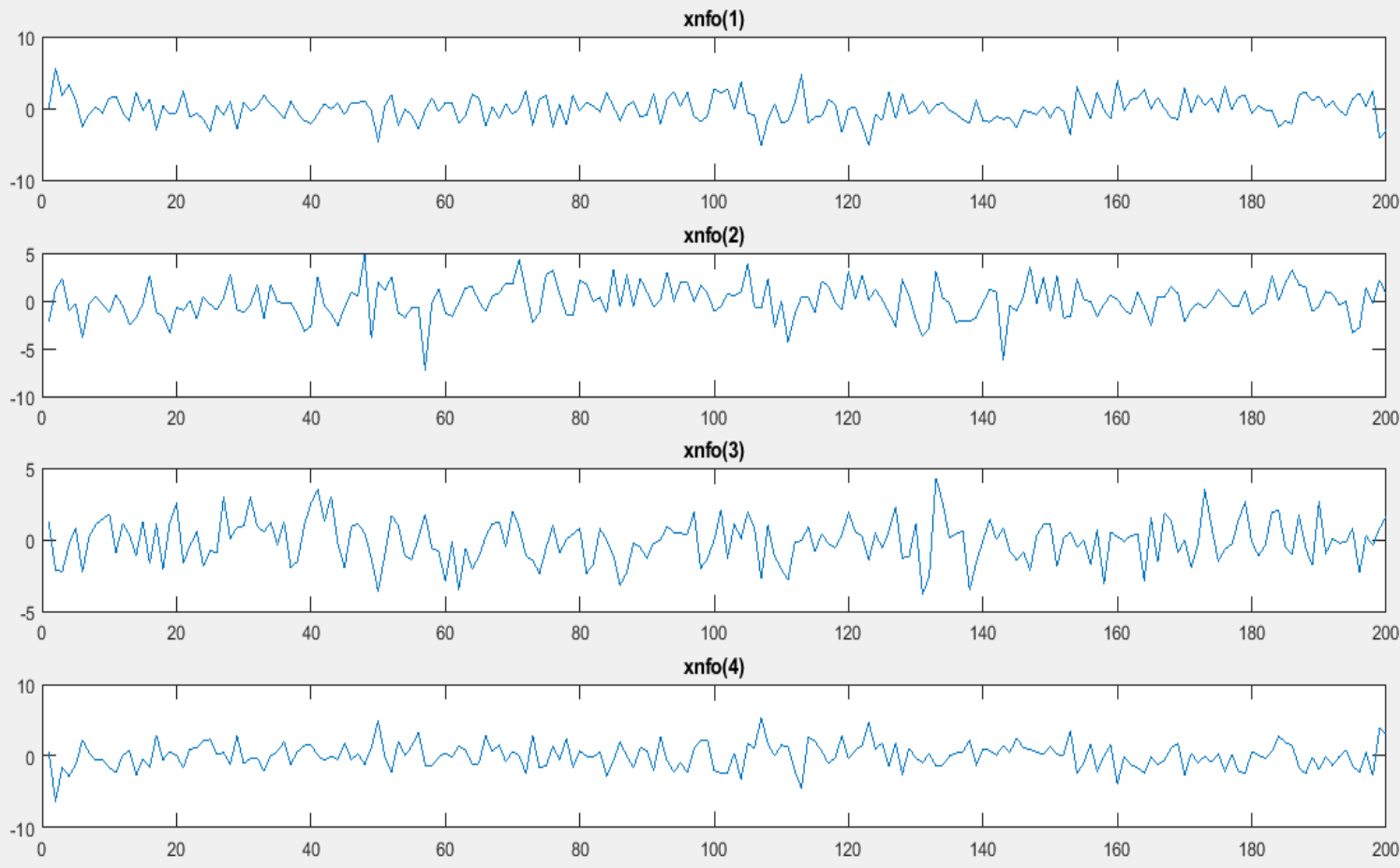

Figure 4. The Source Signal with Steering vector

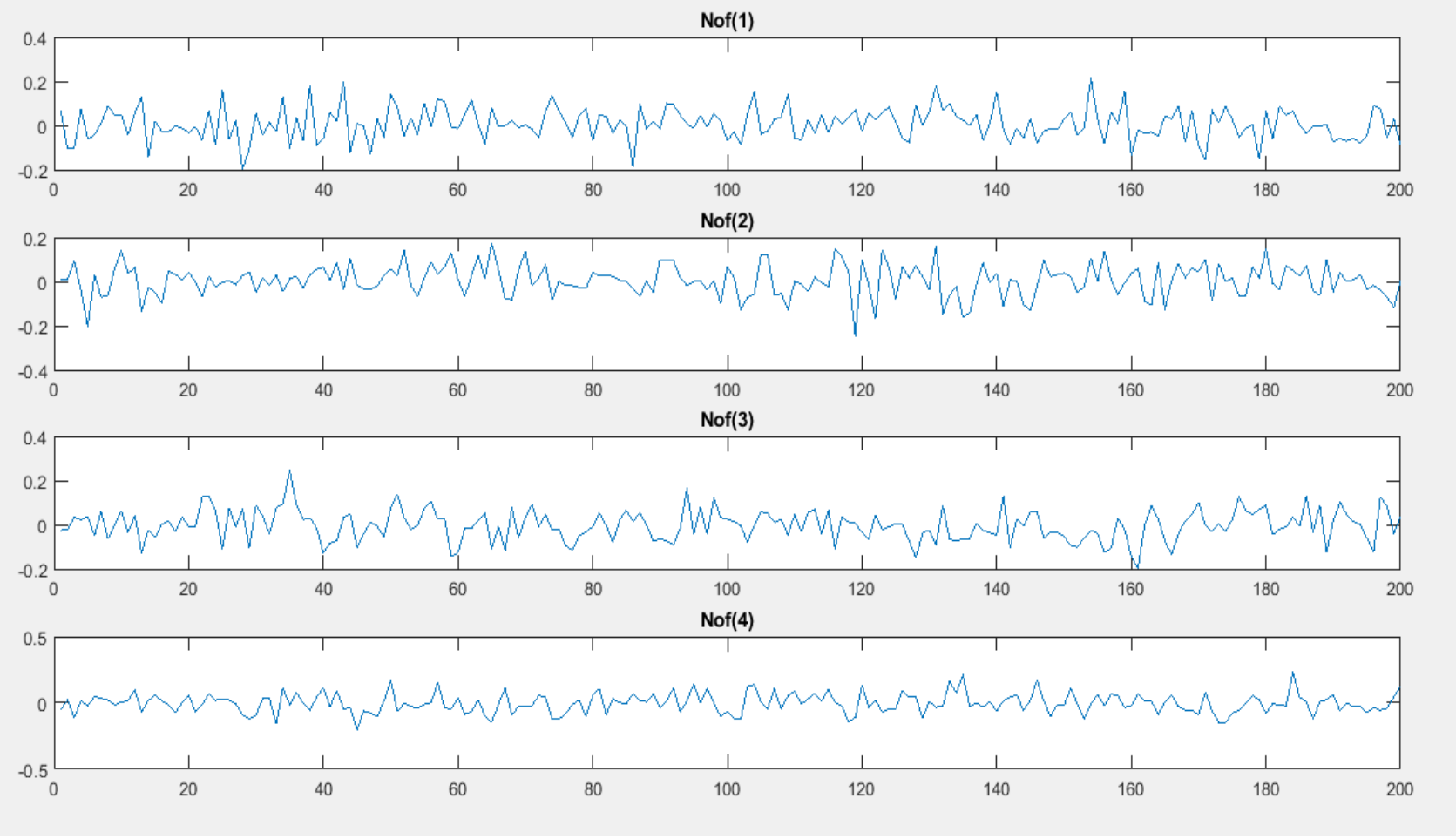

Figure 5. Noise Signals 


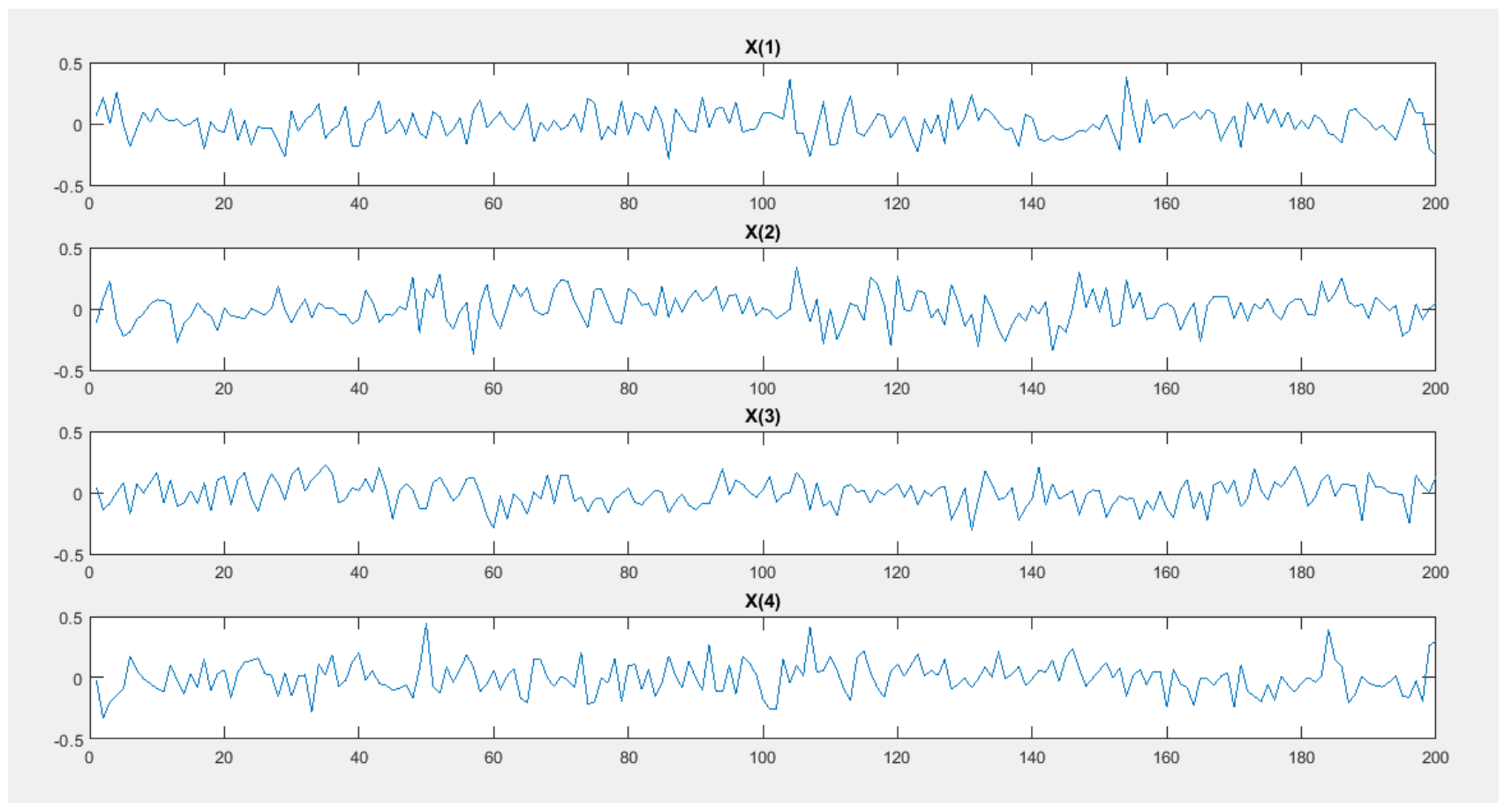

Figure 6. The Incident Signal with noise

The mixed multikernel implementation carried out for the DOA estimation gives a better DOA estimation results as shown in Figure 7. It can be observed that the estimated
AOA are accurate to that the source signals are generated as shown in the Table1.

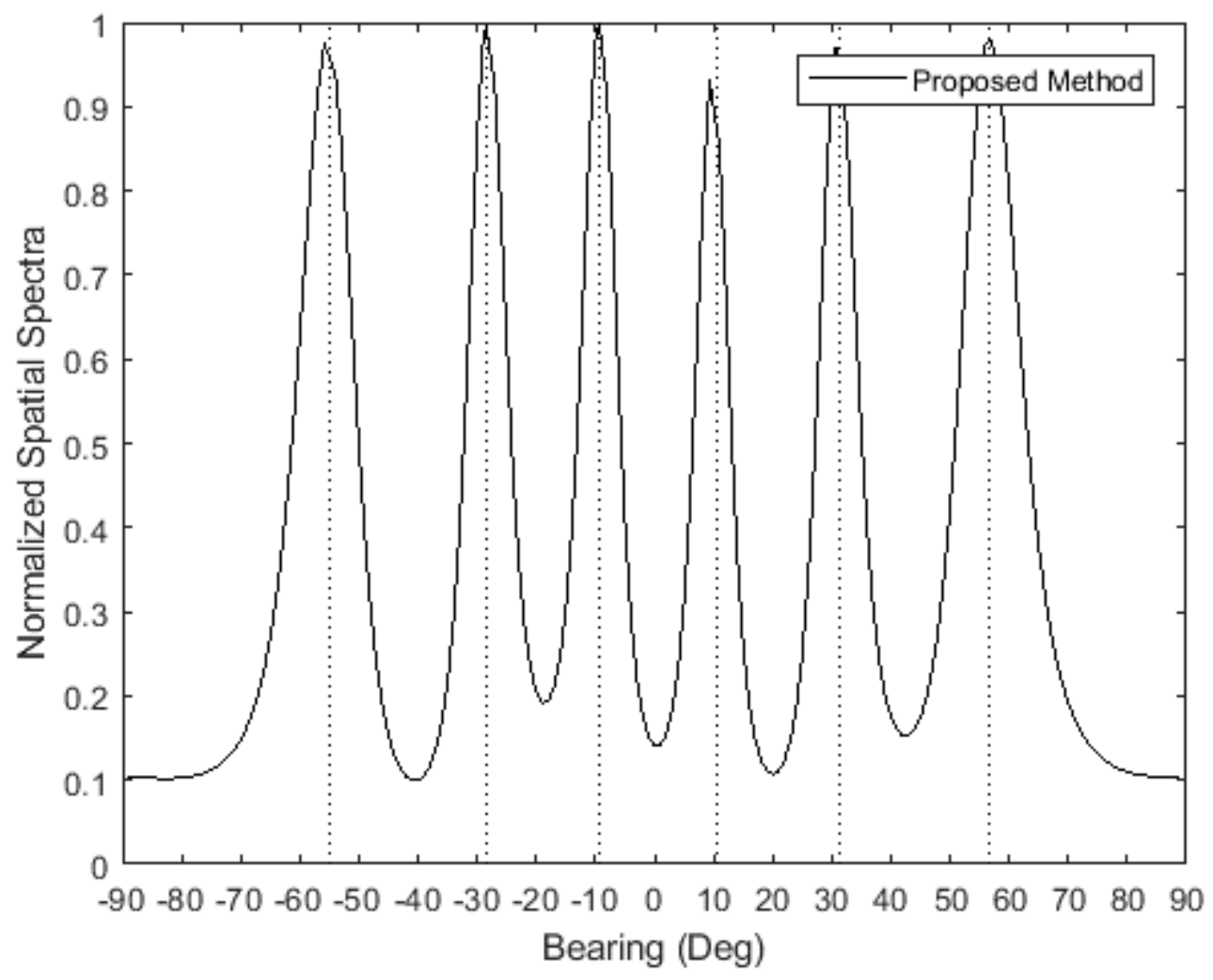

Figure 7. Spatial Spectra Depicting DOAs at the peak (dotted lines) from the mixed model Multikernel implementation 
Figure 8. It is observed that the convergence of the BPDN algorithm thus proposed is satisfactory.

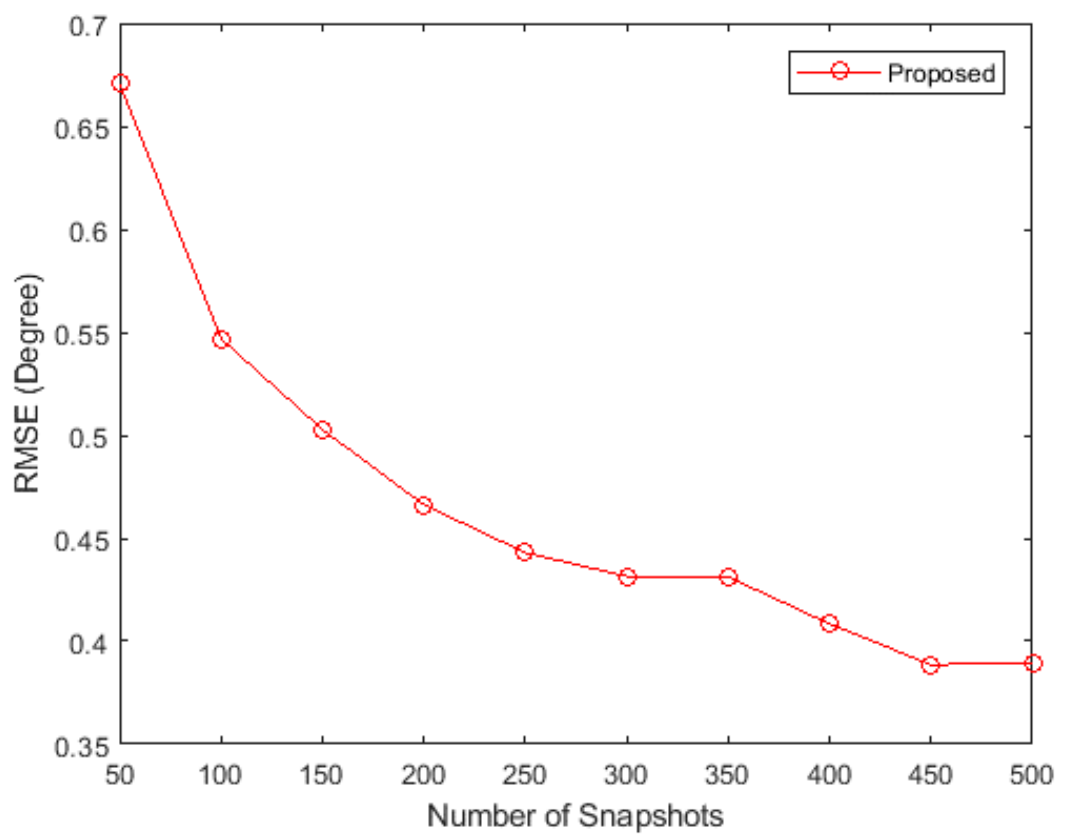

Figure 2. Number of Snapshots Vs RMSE with SNR $=0$

The results from the above experiment defines that there is a better source localization possible with the proposed mixed model multikernel BPDN approach. The numerical simulations thus carried out has provided a better results compared to the previous implementation.

\section{CONCLUSION}

The Matlab based mixed model multikernel based BPDN algorithm is used to estimate the DOA in the non-uniform array of antennas. It is found that the results obtained for the DOA estimation is better compared to the previous implementations. The accuracy of DOA estimation is found to be efficient along with the better RMSE reduction and better convergence is observed. The Matlab based simulation results suggests that the hardware realization of this proposed algorithm is feasible.

\section{REFERENCES}

1. Nan $\mathrm{Hu}$, Bing Sun, Jiajun Wang, Jisheng Dai, Chunqi Chang, "Source localization for sparse array using nonnegative sparse Bayesian learning", Signal Processing, v.127 n.C, p.37-43, October 2016

2. Bishop C (2006) Pattern recognition and machine learning. Springer, Berlin

3. McLachlan G, Peel D (2000) Finite mixture models. Wiley, New York

4. Schmolck A, Everson R (2007) Smooth relevance vector machine: a smoothness prior extension of the RVM. Mach Learn 68(2):107-135

5. Seeger M (2008) Bayesian inference and optimal design for the sparse linear model. J Mach Learn Res 9:759-813

6. Shi J, Wang B (2008) Curve prediction and clustering with mixtures of Gaussian process functional regression models. Stat Comput 18:267-283

7. Zhong M (2006) A variational method for learning sparse Bayesian regression. Neurocomputing 69:2351-2355

8. Gonen M, Alpaydin E (2011) Multiple kernel learning algorithms. J Mach Learn Res 12:2211-2268

9. R.T. Hoctor, S.A. Kassam, "The unifying role of the co-array in aperture synthesis for coherent and incoherent imaging", Proceedings of the IEEE, vol.78, pp. 735-752,1990.

10. David.P. Wipf, Bhaskar D. Rao, “ An empirical Bayesian strategy for solving the simultaneous sparse approximation problem", IEEE Transactions on Signal Processing, vol. 55(7), pp. 3704-3716,2007
11. Zhilin Zhang, Bhaskar D. Rao, "Sparse signal recovery with temporally correlated source vectors using sparse Bayesian learning", IEEE Journal of Selected Topics in Signal Processing, vol. 5(5) pp. 912-926, 2011.

12. Yanning Shen, et al, "Pattern-coupled sparse Bayesian learning for recovery of block-sparse signals", 2014 IEEE International Conference on Acoustics, Speech and Signal Processing, vol. 63(2), pp.360-37, 2015 .

13. Jun Fang, et al, "Two-dimensional pattern-coupled sparse Bayesian learning via generalized approximate message passing", IEEE Transactions on Image Processing, vol.25(6), pp.2920-2930, 2016.

14. Zai Yang, et al, "Off-grid direction of arrival estimation using sparse Bayesian inference", IEEE Transactions on Signal Processing, vol.61(1), pp.38-43,2013.

15. "Sparse Array Signal Processing: New Array Geometries, Parameter Estimation, and Theoretical Analysis" Doctorate Thesis by Chun-Lin Liu, https://thesis.library.caltech.edu/10970/

16. Wing-Kin Ma, et al, "DOA estimation of quasi-stationary signals with less sensors than sources and unknown spatial noise covariance: A Khatri-Rao subspace approach", IEEE Transactions of. Signal Processing, vol. 58(4),pp. 2168-2180, 2010.

17. B. Ottersten et al, "Covariance matching estimation techniques for array signal processing applications", Digit. Signal Processing, vol.8(3), pp.185-210,1998.

18. Konstantinos Blekas "Sparse regression mixture modeling with the multi-kernel relevance vector machine” Knowl Inf Syst (2014) 39: 241. https://doi.org/10.1007/s10115-013-0704-0

19. S. Derin Babacan, et al, "Bayesian compressive sensing using Laplace priors", IEEE Transactions of Image Processing,vol. 19(1) pp. $53-63,2010$ 